\title{
Fast generation of spin-squeezed states in bosonic Josephson junctions
}

\author{
B. Juliá-Díaz, ${ }^{1}$ E. Torrontegui, ${ }^{2}$ J. Martorell, ${ }^{3}$ J. G. Muga, ${ }^{2,4}$ and A. Polls ${ }^{3}$ \\ ${ }^{1}$ ICFO-Institut de Ciències Fotòniques, Parc Mediterrani de la Tecnologia, 08860 Barcelona, Spain \\ ${ }^{2}$ Departamento de Química-Física, UPV-EHU, Apartado 644, 48080 Bilbao, Spain \\ ${ }^{3}$ Departament d'Estructura i Constituents de la Matèria, Facultat de Física, U. Barcelona, 08028 Barcelona, Spain \\ ${ }^{4}$ Department of Physics, Shanghai University, 200444 Shanghai, People's Republic of China \\ (Received 5 July 2012; revised manuscript received 18 September 2012; published 19 December 2012)
}

\begin{abstract}
We describe methods for the fast production of highly coherent-spin-squeezed many-body states in bosonic Josephson junctions. We start from the known mapping of the two-site Bose-Hubbard (BH) Hamiltonian to that of a single effective particle evolving according to a Schrödinger-like equation in Fock space. Since, for repulsive interactions, the effective potential in Fock space is nearly parabolic, we extend recently derived protocols for shortcuts to adiabatic evolution in harmonic potentials to the many-body BH Hamiltonian. A comparison with current experiments shows that our methods allow for an important reduction in the preparation times of highly squeezed spin states.
\end{abstract}

DOI: 10.1103/PhysRevA.86.063623

PACS number(s): 03.75.Kk, 42.50.Dv, 05.30.Jp, 42.50.Lc

\section{INTRODUCTION}

Recent experimental results from the groups of Oberthaler and Treutlein have provided compelling evidence of the generation of spin-squeezed states in bosonic Josephson junctions [1-4]. Two different setups with ultracold ${ }^{87} \mathrm{Rb}$ bosons have been explored: (a) a cloud trapped in an external double-well potential [1,5,6] [external bosonic Josephson junctions (BJJ)] and (b) a cloud of atoms in two different hyperfine states trapped in a single harmonic potential with a linear coupling between the two internal states [2-4] (internal BJJ). In both the internal and external Josephson junctions, the experimental setups were able to reproduce to a large extent the well-known two-site Bose-Hubbard (BH) model [6-8], a Lipkin-Meshkov-Glick-like (LMG) Hamiltonian [9]. In this way, the experiments confirmed the predicted existence of strongly squeezed spin states [10] and, correspondingly, of large sets of entangled pseudospins [11,12].

Simultaneously, and due to the need to control and implement quantum resources, there has been an increasing interest in developing fast protocols to shortcut adiabatic following $[13,14]$. The key purpose is to engineer procedures to drive, in a finite time, a system from some initial state to a final state that could be reached with an adiabatic, slow process. The different protocols may, in addition, aim at minimizing the transient energy excitations, reducing the sensitivity to noise, or minimizing other variables of interest [15]. It should be noted that, in general, the initial and final states are not necessarily required to be the ground states (GSs) of the system. In this work, however, we concentrate on transitions between ground states corresponding to different values of the model parameters which can be controlled externally. The proposed method is designed so that the desired final state is produced as a stationary eigenstate of the Hamiltonian, with no need to freeze the dynamics. Analytical formulas to perform this type of process exist for the harmonic oscillator [13].

In this article we demonstrate that these methods can also be employed to produce the highly correlated many-body quantum states described by the BH Hamiltonian, such as squeezed states, broadening the current state of the art which has so far dealt only with condensed cold gases [16]. To do so, we benefit from the explicit mapping, in the large $N$ limit, between the two-site Bose-Hubbard model and an effective single-particle system described by a Schrödinger-like equation [17-19]. ${ }^{1}$ As will be shown, this connection allows us to use methods which were originally developed for single-particle dynamics, to shortcut the adiabatic following in a many-body problem. As a figure of merit, the methods proposed would allow one to prepare highly number-squeezed states in roughly half the time needed in the experimental setup of Ref. [3].

Our proposal presents important differences with respect to the recent work of Ref. [22]. We target the formation of spin-squeezed states, while [22] uses optimal control theory (OCT) to produce catlike states. In our method, the final state of the system is the ground state, with no need to stop or freeze the dynamics, while in Ref. [22] a stoppage is required once the desired state is reached. Also, as detailed below, our method to shortcut the adiabatic evolution is very robust, only requiring high precision control during the initial and final times.

The article is organized as follows. In Sec. II we present the Bose-Hubbard model and introduce the semiclassical $1 / N$ approximate model. In Sec. III we present the methods to shortcut the adiabatic evolution and adapt them to our specific problem. In Sec. IV we discuss the obtained results. Finally, Sec. V presents our conclusions.

\section{DESCRIPTION OF THE MODEL}

We assume that in the BJJ, the system of ultracold bosons is well modeled by the Bose-Hubbard Hamiltonian $\hbar \mathcal{H}_{\mathrm{BH}}$,

$$
\mathcal{H}_{\mathrm{BH}}=-2 J \hat{J}_{x}+U \hat{J}_{z}^{2},
$$

where the pseudoangular momentum operator $\hat{\mathbf{J}} \equiv\left\{\hat{J}_{x}, \hat{J}_{y}, \hat{J}_{z}\right\}$ is defined as

$$
\begin{aligned}
& \hat{J}_{x}=\frac{1}{2}\left(\hat{a}_{1}^{\dagger} \hat{a}_{2}+\hat{a}_{2}^{\dagger} \hat{a}_{1}\right), \quad \hat{J}_{y}=\frac{1}{2 i}\left(\hat{a}_{1}^{\dagger} \hat{a}_{2}-\hat{a}_{2}^{\dagger} \hat{a}_{1}\right), \\
& \hat{J}_{z}=\frac{1}{2}\left(\hat{a}_{1}^{\dagger} \hat{a}_{1}-\hat{a}_{2}^{\dagger} \hat{a}_{2}\right),
\end{aligned}
$$

\footnotetext{
${ }^{1}$ In the context of the LMG model, this connection was established earlier [20,21], albeit with no known relation at the time with ultracold atom physics.
} 
where $\hat{a}_{j}^{\dagger}$ creates a boson in site $j$, and $\left[\hat{a}_{i}, \hat{a}_{j}^{\dagger}\right]=\delta_{i, j}$. $J$ is the hopping strength, taken positive, and $U$ is the nonlinear coupling strength proportional to the atom-atom $s$-wave scattering length. ${ }^{2}$ In this work we consider repulsive interactions, $U>0$. For internal BJJ, the interspecies $s$-wave scattering length in ${ }^{87} \mathrm{Rb}$ atoms can be varied by applying an external magnetic field thanks to a well-characterized Feshbach resonance at $B=9.1 \mathrm{G}$, as done in Ref. [4] for the setup that we are considering, thus permitting precise control over the $U$. In this work, we use a time-dependent $U(t)$, keeping $J$ and $N$ fixed during the time evolution. This has been experimentally achieved already in an atom-chip experiment [3]. In the experiments of the Heidelberg group one would need to track the resonance of the linear coupling (responsible for the hopping term in the Hamiltonian) which is altered due to the second-order Zeeman shift when $B$ is varied. Albeit technically challenging, this is within reach of the current experimental setups [23].

The time-dependent Schrödinger equation (TDSE) is written as

$$
\imath \partial_{t}|\Psi\rangle=\mathcal{H}_{\mathrm{BH}}|\Psi\rangle
$$

For a given $N$, an appropriate many-body basis for this bosonic system is the Fock basis, $\left\{\left|m_{z}=\left(N_{1}-N_{2}\right) / 2\right\rangle\right\}$, with $m_{z}=$ $-N / 2, \ldots, N / 2$. A general many-body state $|\Psi\rangle$ can then be written as

$$
|\Psi\rangle=\sum_{m_{z}=-N / 2}^{N / 2} c_{m_{z}}\left|m_{z}\right\rangle .
$$

As customary, the number squeezing parameter is defined as Refs. [1,24]

$$
\xi_{N}^{2}(t)=\frac{\Delta \hat{J}_{z}^{2}}{\left(\Delta \hat{J}_{z}^{2}\right)_{\text {bin }}},
$$

where $\Delta \hat{J}_{z}^{2} \equiv\left\langle\hat{J}_{z}^{2}\right\rangle-\left\langle\hat{J}_{z}\right\rangle^{2}$ and $\left(\Delta \hat{J}_{z}^{2}\right)_{\text {bin }}=N / 4$ for a coherent state with $\left\langle\hat{J}_{z}\right\rangle=0$. The many-body state is said to be number squeezed if $\xi_{N}<1$ [10]. The coherent spin-squeezing parameter, which accounts for reductions in the fluctuations in $\hat{J}_{z}$, taking $\left\langle\hat{J}_{y}\right\rangle=0$, is defined as Refs. [10,11,24]

$$
\xi_{S}^{2}=\frac{N\left(\Delta \hat{J}_{z}^{2}\right)}{\left\langle\hat{J}_{x}\right\rangle^{2}}=\frac{\xi_{N}^{2}}{\alpha^{2}},
$$

where the phase coherence of the many-body state is $\alpha(t)=$ $\left\langle\Psi(t)\left|2 \hat{J}_{x} / N\right| \Psi(t)\right\rangle . \xi_{S}$ takes into account the delicate compromise between improvements in number squeezing and loss of coherence. States with $\xi_{S}<1$ have been proposed to be used in a new Ramsey-type atom interferometer with an increased phase precision compared to the coherent spin state [24]. This gain in precision can be directly related to entanglement in the system [25].

As detailed in Refs. [17-19] a systematic expansion of $\mathcal{H}_{N} \equiv \mathcal{H}_{\mathrm{BH}} /(N J)$ in powers of $h \equiv 1 / N$ gives in the semiclassical limit $N \gg 1$, the pseudo-Schrödinger-like

\footnotetext{
${ }^{2}$ In the internal BJJ, $U$ is proportional to $a_{1,1}+a_{2,2}-2 a_{1,2}$, with $a_{1,1}$ and $a_{2,2}$ the intraspecies scattering lengths and $a_{1,2}$ the interspecies one [4].
}

equation

$$
\mathcal{H}_{N}(z) \psi(z) \equiv-2 h^{2} \partial_{z} \sqrt{1-z^{2}} \partial_{z} \psi(z)+\mathcal{V}(z) \psi(z),
$$

where

$$
\mathcal{V}(z)=-\sqrt{1-z^{2}}+\frac{1}{2} \Lambda z^{2}
$$

$z=m_{z} /(N / 2), \Lambda=N U /(2 J)$, and $\psi(z)=\sqrt{N / 2} c_{m_{z}}$, normalized as $\int_{-1}^{1} d z|\psi(z)|^{2}=1$. The corresponding TDSE can be written as

$$
i h \partial_{t} \psi(z, t)=\mathcal{H}_{N} \psi(z, t)
$$

with time measured in units of $1 / J$. In these units the Rabi time is defined as $t_{\text {Rabi }}=\pi / J$. Let us emphasize that we construct a systematic expansion on the small parameter $h$ but we do not take the formal limit $h \rightarrow 0$. The validity of the expansion will improve as the considered number of atoms is increased, but it is already accurate for $N \gtrsim 50$.

As explained in Ref. [19], and previously noted by other authors $[17,26]$, for repulsive interactions the potential in Fock space, $\mathcal{V}(z)$, is to a very good approximation a harmonic oscillator: Neglecting the $z$ dependence of the effective mass term and expanding $\sqrt{1-z^{2}} \simeq 1-z^{2} / 2$ in $\mathcal{V}(z)$, Eq. (7), reduces to

$$
\mathcal{H}_{N} \simeq-2 h^{2} \partial_{z}^{2}+\frac{1}{8} \omega^{2} z^{2}
$$

with $\omega=2 \sqrt{1+\Lambda}$.

We have checked that all the expectation values of $\hat{J}_{i}$ and $\hat{J}_{i}^{2}, i=x, y, z$ computed by directly solving the TDSE for the BH Hamiltonian equation (3), with $\Lambda \equiv \Lambda(t)$ as required by the control protocols explained below, and the corresponding approximate ones using the solution Eq. (7) together with the explicit expressions given in Ref. [19] agree almost perfectly in all calculations reported in this article. The only minor discrepancy between the results obtained using the continuous version, Eq. (7), and the full TDSE for the Bose-Hubbard Hamiltonian, Eq. (3), is explicitly shown in Fig. 3.

It is worth stressing that Eq. (7) allows one to study quantum properties, i.e., squeezing, which are beyond the usual fully classical description [27]. Setting $h=0$ in Eq. (7), which removes the kinetic term in Fock space, one obtains Eq. (5) of Ref. [27] around $\phi=0$.

\section{FAST SHORTCUT TO ADIABATIC EVOLUTION}

Our method for fast adiabaticlike preparation of a given ground state requires control of the atom-atom interaction at time scales of fractions of the Rabi time. In what follows we make use of Eq. (10) to find an optimal solution for the control parameter $\Lambda(t)$ with our shortcut (SC) method (explained below), and we later use Eq. (3) with $\Lambda(t)$ given by the solution found, to compute the squeezing, visibility, and the other parameters presented in Figs. 1-4. In the parabolic approximation discussed above, the protocol developed in Ref. [13] has a direct translation into our variables. We need to impose the following time variation of $\Lambda,{ }^{3}$

\footnotetext{
${ }^{3}$ Note that the derivations in the previous section imply a fixed $J$ and $N$, thus the variation in $\Lambda$ has to be due to a variation in $U$.
} 


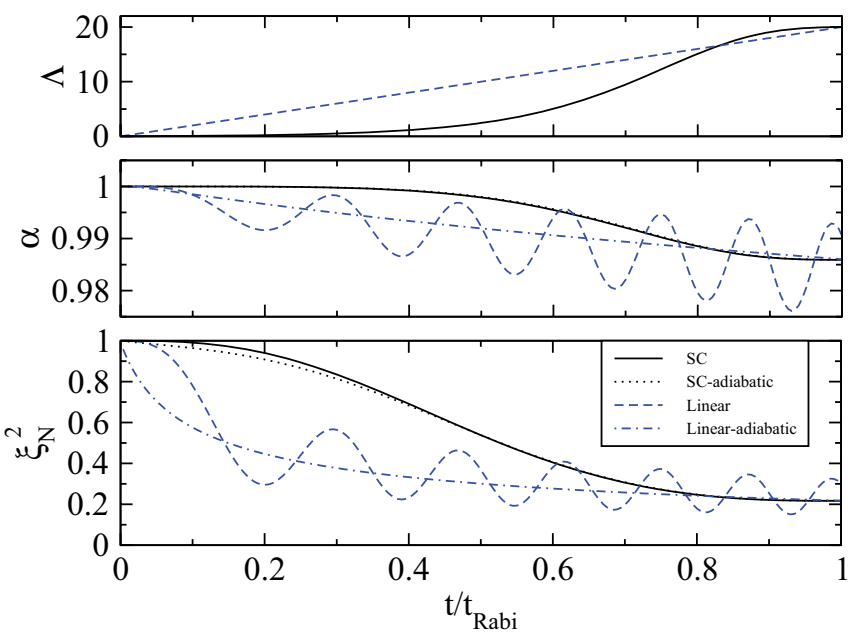

FIG. 1. (Color online) Time evolution of the coherence $\alpha(t)$ and the number squeezing $\xi_{N}^{2}$ of the system obtained solving the TDSE for the BH, Eq. (3), using the fast adiabatic evolution of Eq. (11) (solid) compared to the case of linear ramping of $\Lambda(t)$ (dashed). The corresponding instantaneous adiabatic results for both the fast adiabatic case and a linear ramping are given in dotted and dot-dashed, respectively. $\Lambda(0)=0, \Lambda\left(t_{\text {Rabi }}\right)=20$, and $N=100$.

$\Lambda(t)=\omega^{2}(t) / 4-1$, where $\omega^{2}(t)$ obeys the Ermakov equation,

$$
\ddot{b}(t) / J^{2}+\omega^{2}(t) b(t)=\omega_{0}^{2} / b^{3}(t),
$$

with time in units of $1 / J$ as explained above. The key ingredient is to ensure that $b(t)$ satisfies the six frictionless conditions:

$$
\begin{aligned}
& b(0)=1, \quad b\left(t_{F}\right)=r, \\
& \dot{b}(0)=\ddot{b}(0)=\dot{b}\left(t_{F}\right)=\ddot{b}\left(t_{F}\right)=0,
\end{aligned}
$$

where $r=\sqrt{\omega_{0} / \omega_{F}}, \omega_{0}=2 \sqrt{1+\Lambda_{0}}$, and $\omega_{F}=2 \sqrt{1+\Lambda_{F}}$. The fact that these can be fulfilled by an infinite set of $b(t)$ has two important consequences: (1) This freedom allows one to apply tools of OCT to produce $b(t)$ 's which ensure fidelity 1 and satisfy other constraints. For instance: (a) optimize the value of $t_{F}$ (in contrast, e.g., with the simple "bang-bang" methods described in the Appendix); (b) ensure that $\Lambda(t)$ is bound by previously chosen experimental values; or (c) guarantee that the potential energy of the system at intermediate steps is bound by some desired value $[15,28]$. (2) The method is extremely robust: In an experimental realization aiming at a final fidelity equal to 1 , the success is guaranteed provided the frictionless conditions are satisfied, with no need to have a precise control during the intermediate evolution [16]. This is an important advantage with respect to other protocols, e.g., the ones considered in Ref. [22]. To give an analytic and smooth example we consider here the polynomial ansatz for $b(t)$ from Ref. [13],

$$
b(t)=6(r-1) s^{5}-15(r-1) s^{4}+10(r-1) s^{3}+1,
$$

with $s=t / t_{F}$. A brief comparison to another ansatz is presented below.

\section{RESULTS}

Our calculations confirm that our method, although not exact, is extremely accurate in the many-body simulations despite the fact that it was derived for the single-particle problem in a harmonic oscillator Hamiltonian. This is due to the validity of the explained mapping of the $\mathrm{BH}$ to the single-particle pseudo-Schrödinger equation, which turns out to be well approximated by a harmonic oscillator potential. This harmonic approximation improves as $N$ is increased because the spread of the GS wave function is $\sim 1 / \sqrt{N}$, thus exploring only the very central part of $\mathcal{V}(z)$ in $z \in[-1,1]$. Exceptions appear in the ultrafast preparation as discussed later.

Let us start by comparing the fast protocol to shortcut the adiabatic following presented above to the case of a linear ramping $\Lambda_{l}(t)=\Lambda(0)+\left(t / t_{F}\right)\left[\Lambda\left(t_{F}\right)-\Lambda(0)\right]$. In this case we consider a relatively long final time of one full Rabi period $t_{\text {Rabi }}=\pi / J$ (see Fig. 1). For both $\Lambda(t)$ we present the results of the corresponding instantaneous ground state of the many-body system for comparison. First, we note that the $\mathrm{SC}$ does produce a final result which has the same coherence $\alpha$ and coherent number squeezing $\xi_{N}^{2}$ as the corresponding adiabatically evolved case. In contrast, the linearly ramped system deviates notably from the adiabatically evolved state at the final time. As we are considering fairly long times, the $\mathrm{SC}$ is not found to deviate appreciably from the instantaneous adiabatic following of the state, except for a short period at early times. The transient nonadiabaticity will be shown to increase as we require shorter $t_{F}$ 's.

The fidelity between the evolved many-body state $|\Psi(t)\rangle$ and the corresponding instantaneous ground state $\left|\left\langle\Psi(t) \mid \Psi_{\mathrm{GS}}\right\rangle\right|$ is plotted in Fig. 2 and is seen to be extremely close to 1 at the final time for both $t_{F}=0.2 t_{\text {Rabi }}$ and $1 t_{\text {Rabi }}$. In the figure we compare the full many-body evolution to the corresponding instantaneous ground state for different $t_{F}$. As mentioned above, for short times, the fast-adiabatic passage produces intermediate many-body states which depart from the instantaneous ones, as seen clearly in the drop of the fidelity for very short times in the $t_{F}=0.2 t_{\text {Rabi }}$ case.

The freedom in choosing $b(t)$ is large, providing a very robust protocol when the frictionless conditions are satisfied. For example, the use of a nonpolynomial choice ${ }^{4}$ for $b(t)$ still produces a fidelity equal to 1 at $t=t_{F}$ [see dot-dashed lines in Figs. 2(a) and 2(b)]. This freedom can be exploited, e.g., to fulfill actual experimental constraints on the control parameters.

\section{A. Ultrashort adiabatic production of squeezed states}

As described in Ref. [13] for a particle in a harmonic oscillator and ultrafast adiabatic evolution, the method presented requires a certain period of the evolution where the trapping potential is actually reversed into an expelling potential (an inverted parabola). Through the previously explained mapping, this corresponds in our many-body $\mathrm{BH}$ problem to

\footnotetext{
${ }^{4}$ We consider for illustration, $b(t)=r^{6 s^{5}-15 s^{4}+10 s^{3}}$.
} 

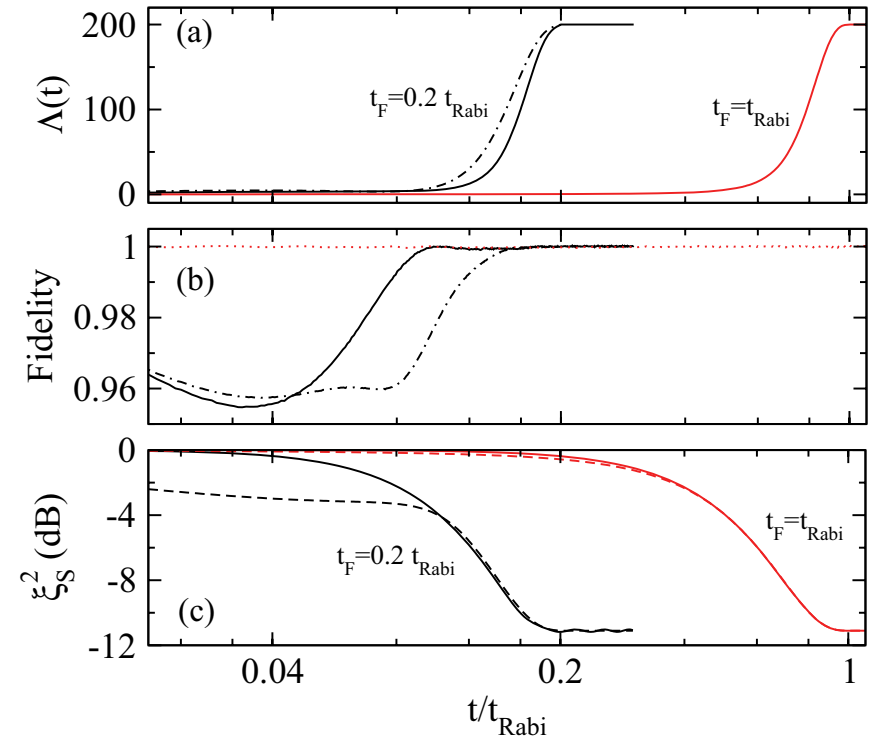

FIG. 2. (Color online) (a) Considered $\Lambda(t)$ : two final times are considered, $t_{F}=0.2 t_{\text {Rabi }}$ and $1 t_{\text {Rabi }}$. (b) Fidelity for the cases considered; note for $t_{F}=t_{\text {Rabi }}$ the curve is $\sim 1$ during all the intervals. The dot-dashed line in panels (a) and (b) corresponds to a calculation using a nonpolynomial ansatz for $b(t)$ (see text). (c) Coherent spin squeezing $\xi_{S}^{2}$ as a function of $t$ using the fast-adiabatic process described in the text. The adiabatic $\xi_{S}^{2}$, corresponding to the instantaneous ground state for each $\Lambda$, is shown in dashed lines in the lower panel. The initial and final $\Lambda$ are 0 and 200. $N=100$.

a period of time where the atom-atom interaction is switched from repulsive to attractive, $\Lambda<0$. For attractive interactions the potential $\mathcal{V}(z)$ becomes a double-well potential in Fock space and the parabolic approximation of Eq. (10) only holds for central regions in $z$ [19]. Thus, one can expect that the present method should still work only when the wave function $\psi(z, t)$ does not spread in $z$ significantly during the time where the atom-atom interactions are attractive. As seen in Fig. 3 this is indeed the case. We consider $t_{F}=0.05 t_{\text {Rabi }}, \Lambda(0)=0$, and $\Lambda\left(t_{F}\right)=500$. Then the required $\Lambda(t)$ (upper panel) becomes negative for $0.025 \lesssim t / t_{\text {Rabi }} \lesssim 0.04$. The method is seen to work accurately, producing a fidelity $\sim 1$ at the final time. The intermediate evolution is, however, highly diabatic, with close to zero fidelity between the evolved state and its corresponding adiabatic one, during the $\Lambda(t)<0$ evolution. The effect of a systematic error in the implementation is analyzed in Fig. 3 finding that a $10 \%$ deviation in the value of $\Lambda(t)$ results in a decrease of the fidelity to $\sim 0.87$, preserving $\xi_{S}^{2} \sim-12 \mathrm{~dB}$ at $t_{F}$.

\section{B. Comparison to existing experimental setups}

To illustrate the improvement in preparation times which can be obtained with the protocols discussed above, we consider some characteristic parameter values which have already been implemented experimentally. In the experiment of Gross et al. [2], the ultracold atomic cloud is formed by $\sim 400$ atoms of ${ }^{87} \mathrm{Rb}$. The nonlinear parameter achieved is $U=$ $2 \pi \times 0.063 \mathrm{~Hz}$, while the linear coupling $2 J$ can be varied from $0 \mathrm{~Hz}$ to $2 \pi \times 600 \mathrm{~Hz}$. With these conditions, a number squeezing of $\xi_{N}^{2} \sim-8.2 \mathrm{~dB}$ was achieved after $\sim 20 \mathrm{~ms}$.
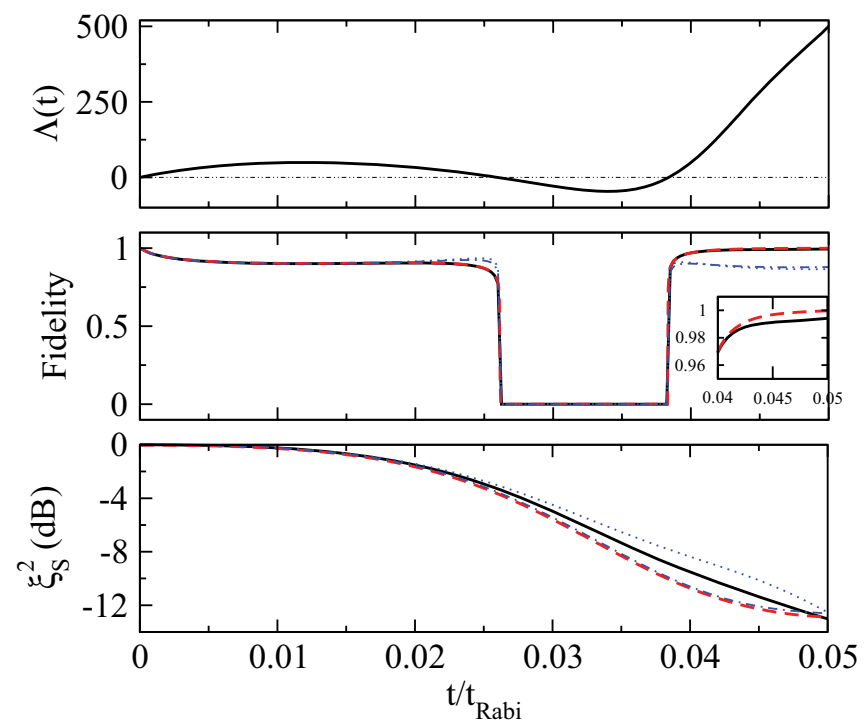

FIG. 3. (Color online) Considered $\Lambda(t)$ (upper panel), fidelity (middle panel), and coherent spin squeezing $\xi_{S}^{2}$ (lower panel) as a function of $t$ for the exact Bose-Hubbard calculation (solid) and for the Schrödinger-like equation (7) (dashed, almost overlapped with the solid in the upper and middle panels). The final time $t_{F}$ is $0.05 t_{\text {Rabi }}$. The initial and final $\Lambda$ are 0 and 500. The dotted and dot-dashed lines are obtained assuming the actual $\Lambda(t)$ is $10 \%$ smaller or larger than the nominal one, respectively. $N=100$. The inset zooms on the times close to $t_{F}$.

Let us consider a Rabi coupling $2 J=2 \pi \mathrm{Hz}\left(t_{\mathrm{Rabi}}=\right.$ $1 \mathrm{~s})$, and the same final value of the nonlinear term $U_{F}=$ $2 \pi \times 0.063 \mathrm{~Hz}$. Assuming an initially noninteracting system $U_{i}=0 \mathrm{~Hz}$, and a control over $U(t)$ from the initial to the final value as required by our protocol, we find that the final value of the adiabatic number squeezing is $\xi_{N}^{2} \sim-8.55 \mathrm{~dB}$. To obtain such a highly number-squeezed state in half the time taken in the experiment of Gross et al., we need $t_{F}=10 \mathrm{~ms}$, which corresponds to $t_{F}=0.005 t_{\text {Rabi }}$. This is achievable with our protocol requiring an ultrafast protocol such as the one presented in Fig. 3.

In the experiment of Riedel et al. [3], the authors trap 1250 atoms with an effective value of the nonlinear coupling $U_{e}=0.49 \mathrm{~Hz}$. They obtain a best value of $\xi_{S}^{2} \sim-2.5 \mathrm{~dB}$ after a time of $\sim 15 \mathrm{~ms}$. Using their values, assuming a good control on $U$ from $U=0 \mathrm{~Hz}$ to $U=U_{e}$ and taking a Rabi coupling of $2 J \sim 2 \pi \times 10 \mathrm{~Hz}\left(t_{\mathrm{Rabi}}=0.1 \mathrm{~s}\right)$ we would obtain an adiabatic coherent squeezing of $\xi_{S}^{2} \sim-5.1 \mathrm{~dB}$. To produce this squeezing value in half the time used by the authors of Ref. [3], we need $t_{F}=7.5 \mathrm{~ms}$, which corresponds to $t_{F}=$ $0.075 t_{\text {Rabi }}$. This is similar to the ultrafast cases considered in Fig. 3.

\section{CONCLUSIONS}

We have presented protocols for fast generation of very coherent-spin-squeezed states in bosonic Josephson junctions. The attained squeezing is the one corresponding to the ordinary adiabatic evolution in the case of repulsive atom-atom interactions, but requires much shorter preparation times. Ordinary adiabatic squeezing is known to improve as $N$ 
is increased as $\xi_{S}^{2} \propto N^{-1}$. Thus, practical methods of fastadiabatic driving present important advantages for any future experimental implementation of BJJs where they are used to produced highly squeezed spin states. The present procedures require good control of the time variation of the atom-atom scattering length during the desired period, a possibility now at hand in current experimental setups for BJJ's. The methods have been obtained by extending recently developed protocols for fast-adiabatic evolution originally devised for a single particle in a time-dependent harmonic trap, to the BoseHubbard Hamiltonian. The experimental implementation of the proposal would represent a useful step towards the fast preparation of many-body entangled quantum resources.

\section{ACKNOWLEDGMENTS}

The authors thank M. Oberthaler and T. Zibold for useful comments and discussions. This work has been supported by FIS2008-01661, 2009-SGR1289, IT472-10, FIS2009-12773C02-01, FIS2008-00784 TOQATA, and the UPV/EHU under program UFI 11/55. B.J.-D. is supported by the Ramón y Cajal program. E.T. acknowledges financial support from the Basque Government (Grant No. BFI08.151).

\section{APPENDIX: BANG-BANG METHODS}

Another set of protocols known to produce fast-adiabatic evolution in harmonic oscillator Hamiltonians are the so-called bang-bang methods [29]. The translation of one of the simplest versions of these methods to the present problem requires the following steps assuming that we start as before from an initial ground state corresponding to $\Lambda(0)=\Lambda_{0}$, and want
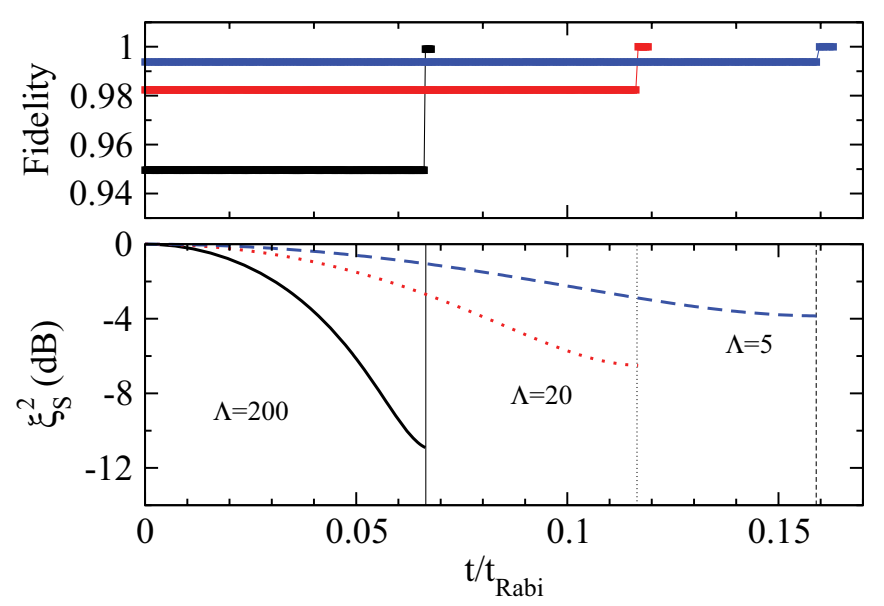

FIG. 4. (Color online) Coherent spin squeezing attainable using a simple bang-bang method as described in the text (lower panel). The fidelity between the evolved state and its corresponding instantaneous evolution is given in the upper panel. $\Lambda(0)=0$ in all cases, while $\Lambda\left(t_{\text {bang }}\right)=5$ (dashed), 20 (dotted), and 200 (solid). $N=100$.

to end up in the ground state corresponding to $\Lambda_{F}$ : (a) at $t=0$ set $\Lambda=\Lambda_{B}=\omega_{0} \omega_{F} / 4-1$ (where $\omega_{0}=\sqrt{1+\Lambda_{0}}$ and $\omega_{F}=\sqrt{1+\Lambda_{F}}$ ), (b) let the system evolve at fixed $\Lambda_{\mathrm{B}}$ until the time $t=t_{B}=t_{\mathrm{Rabi}} /\left(2 \sqrt{\omega_{0} \omega_{F}}\right)$, and (c) change $\Lambda_{B}$ to $\Lambda_{F}$. Note that the final time is not arbitrary, as before, but is fixed by the initial and final values of $\Lambda$. This makes it in practice more unstable with respect to small errors [30]. Under ideal conditions (perfect timing) the method is seen to work well producing fairly low values of $\xi_{S}^{2}$ at times of the order of $t_{\text {Rabi }} / 10$ for $N=100$ atoms with a fidelity of $\sim 1$ at the final time (see Fig. 4).
[1] J. Esteve, C. Gross, A. Weller, S. Giovanazzi, and M. K. Oberthaler, Nature (London) 455, 1216 (2008).

[2] C. Gross, T. Zibold, E. Nicklas, J. Estève, and M. K. Oberthaler, Nature (London) 464, 1165 (2010).

[3] M. F. Riedel, P. Bohi, Y. Li, T. W. Hansch, A. Sinatra, and P. Treutlein, Nature (London) 464, 1170 (2010).

[4] T. Zibold, E. Nicklas, C. Gross, and M. K. Oberthaler, Phys. Rev. Lett. 105, 204101 (2010).

[5] M. Albiez, R. Gati, J. Fölling, S. Hunsmann, M. Cristiani, and M. K. Oberthaler, Phys. Rev. Lett. 95, 010402 (2005).

[6] R. Gati and M. K. Oberthaler, J. Phys. B 40, R61 (2007).

[7] G. J. Milburn, J. Corney, E. M. Wright, and D. F. Walls, Phys. Rev. A 55, 4318 (1997).

[8] A. J. Leggett, Rev. Mod. Phys. 73, 307 (2001).

[9] H. J. Lipkin, N. Meshkov, and A. J. Glick, Nucl. Phys. 62, 188 (1965).

[10] M. Kitagawa and M. Ueda, Phys. Rev. A 47, 5138 (1993).

[11] A. Sørensen, L.-M. Duan, I. Cirac, and P. Zoller, Nature (London) 409, 603 (2001).

[12] J. K. Korbicz, J. I. Cirac, and M. Lewenstein, Phys. Rev. Lett. 95, 120502 (2005).

[13] X. Chen, A. Ruschhaupt, S. Schmidt, A. del Campo, D. GuéryOdelin, and J. G. Muga, Phys. Rev. Lett. 104, 063002 (2010).
[14] X. Chen and J. G. Muga, Phys. Rev. A 82, 053403 (2010); X. Chen, E. Torrontegui, and J. G. Muga, ibid. 83, 062116 (2011); E. Torrontegui, S. Ibañez, X. Chen, A. Ruschhaupt, D. Guéry-Odelin, and J. G. Muga, ibid. 83, 013415 (2011).

[15] X. Chen, E. Torrontegui, D. Stefanatos, Jr-Shin Li, and J. G. Muga, Phys. Rev. A 84, 043415 (2011); E. Torrontegui, X. Chen, M. Modugno, S. Schmidt, A. Ruschhaupt, and J. G. Muga, New J. Phys. 14, 013031 (2012); A. Ruschhaupt, X. Chen, D. Alonso, and J. G. Muga, ibid. 14, 093040 (2012).

[16] J.-F. Schaff, X.-L. Song, P. Vignolo, and G. Labeyrie, Phys. Rev. A 82, 033430 (2010); J.-F. Schaff, X.-L. Song, P. Capuzzi, P. Vignolo, and G. Labeyrie, Europhys. Lett. 93, 23001 (2011).

[17] V. S. Shchesnovich and M. Trippenbach, Phys. Rev. A 78, 023611 (2008).

[18] B. Juliá-Díaz, J. Martorell, and A. Polls, Phys. Rev. A 81, 063625 (2010).

[19] B. Juliá-Díaz, T. Zibold, M. K. Oberthaler, M. Melé-Messeguer, J. Martorell, and A. Polls, Phys. Rev. A 86, 023615 (2012).

[20] A. V. Turbiner, Commun. Math. Phys. 118, 467 (1988).

[21] V. V. Ulyanov and O. B. Zaslavskii, Phys. Rep. 216, 179 (1992).

[22] M. Lapert, G. Ferrini, and D. Sugny, Phys. Rev. A 85, 023611 (2012).

[23] T. Zibold (private communication). 
[24] D. J. Wineland, J. J. Bollinger, W. M. Itano, F. L. Moore, and D. J. Heinzen, Phys. Rev. A 46, R6797 (1992).

[25] L. Pezzé and A. Smerzi, Phys. Rev. Lett. 102, 100401 (2009).

[26] J. Javanainen, and M. Yu. Ivanov, Phys. Rev. A 60, 2351 (1999).

[27] A. Smerzi, S. Fantoni, S. Giovanazzi, and S. R. Shenoy, Phys. Rev. Lett. 79, 4950 (1997).
[28] D. Stefanatos, J. Ruths, and J.-S. Li, Phys. Rev. A 82, 063422 (2010).

[29] P. Salamon, K. H. Hoffmann, Y. Rezek, and R. Kosloff, Phys. Chem. Chem. Phys. 11, 1027 (2009).

[30] E. Torrontegui, X. Chen, M. Modugno, A. Ruschhaupt, D. Guéry-Odelin, and J. G. Muga, Phys. Rev. A 85, 033605 (2012). 